Effects of el ectron temper at ure and density on i on- dust bremsstrahl ung spectrumi n dusty pl asmas

\begin{tabular}{|l|l|}
\hline $\begin{array}{l}\text { jour nal or } \\
\text { publ i cat i on ti t l e }\end{array}$ & Jour nal of Appl i ed Physi cs \\
\hline vol une & 105 \\
\hline page range & 106106 \\
\hline year & 2009- 05-18 \\
\hline URL & ht t p: //hdl . handl e. net $/ 10655 / 1315$ \\
\hline
\end{tabular}




\title{
Effects of electron temperature and density on ion-dust bremsstrahlung spectrum in dusty plasmas
}

\author{
Young-Dae Jung ${ }^{1,2, a)}$ and Izumi Murakami ${ }^{1}$ \\ ${ }^{1}$ National Institute for Fusion Science, Toki, Gifu 509-5292, Japan \\ ${ }^{2}$ Department of Applied Physics, Hanyang University, Ansan, Kyunggi-Do 426-791, South Korea
}

(Received 23 January 2009; accepted 4 April 2009; published online 18 May 2009)

The effects of electron temperature and density on the ion-dust grain bremsstrahlung process in dusty plasmas are studied. The ion-dust bremsstrahlung radiation cross section is obtained as a function of the dust charge, dust radius, Debye length, collision energy, radiation energy, electron density, and electron temperature by using the Born approximation. It is shown that the ion-dust bremsstrahlung radiation cross section decreases with an increase in the electron density in dusty plasmas. It is also shown that the electron temperature suppresses the bremsstrahlung radiation cross section. In addition, the effect of electron temperature on the ion-dust bremsstrahlung process is found to be more significant than the effect of electron density in dusty plasmas. (C) 2009 American Institute of Physics. [DOI: 10.1063/1.3126453]

The bremsstrahlung ${ }^{1-7}$ in plasmas has received much attention since this process is known to be one of the most fundamental processes and the continuum radiation due to the bremsstrahlung process has been a diagnostic tool for investigating the plasma parameters. Recently, there has been a considerable interest in physical processes in plasmas encompassing highly charged dust grains. ${ }^{8-12}$ In addition, it has been shown that the collective dust-plasma interactions are ubiquitous in various astrophysical and laboratory plasmas. ${ }^{10}$ Hence, the collision and radiation processes have been extensively investigated in order to obtain the information on the plasma parameters such as the plasma density and temperature in dusty plasmas. In most cases, the dusty plasmas are consisting of thermal electrons and ions and negatively charged dusty grains, i.e., three-component dusty plasmas. Hence, the ion-dust grain interaction in dusty plasmas would be different from that in only two-component ion-dust plasmas due to the influence of the plasma electrons on dusty plasmas. Thus, in this paper we have investigated the effects of electron density and temperature on the bremsstrahlung process due to the interaction of ion and negatively charged dust grain in three-component dusty plasmas. The ion-dust grain bremsstrahlung radiation cross section is obtained as a function of the dust charge, dust radius, Debye length, collision energy, radiation energy, electron density, and electron temperature by using the Born approximation for the initial and final states of the projectile ion.

In the Born approximation, the differential ion-dust grain bremsstrahlung cross section ${ }^{13} d^{2} \sigma_{b}$ can be obtained by the second-order nonrelativistic perturbation method,

$$
d^{2} \sigma_{b}=d \sigma_{C} d W_{\omega}
$$

where the differential elastic scattering cross section $d \sigma_{C}$ can be represented by

\footnotetext{
${ }^{a)}$ Electronic mail: ydjung@ hanyang.ac.kr.
}

$$
d \sigma_{C}=\frac{1}{2 \pi \hbar v_{0}^{2}}|\bar{V}(\mathbf{q})|^{2} q d q,
$$

$\hbar$ is the rationalized Planck constant, $v_{0}$ is the initial relative collision velocity, $\bar{V}(\mathbf{q})$ is the Fourier transformation of the ion-dust grain interaction potential $V(\mathbf{r})$,

$$
\bar{V}(\mathbf{q})=\int d^{3} \mathbf{r} e^{-i \mathbf{q} \cdot \mathbf{r}} V(\mathbf{r}),
$$

$\mathbf{q}\left(=\mathbf{k}_{0}-\mathbf{k}_{f}\right)$ is the momentum transfer, and $\mathbf{k}_{0}$ and $\mathbf{k}_{f}$ are, respectively, the wave vectors of the initial and final states of the projectile ion. Here, $d W_{\omega}$ represents the differential photon emission probability within the frequency between $\omega$ and $\omega+d \omega$,

$$
d W_{\omega}=\frac{\alpha}{4 \pi^{2}} \Lambda_{i}^{2} \sum_{\hat{\mathbf{e}}}|\hat{\mathbf{e}} \cdot \mathbf{q}|^{2} \frac{d \omega}{\omega} d \Omega,
$$

where $\alpha\left(=e^{2} / \hbar c \cong 1 / 137\right)$ is the fine structure constant, $\Lambda_{i}($ $\left.\equiv \hbar / m_{i} c\right)$ is the Compton wavelength of the ion, $m_{i}$ is the mass of the ion, $c$ is the velocity of the light, $\hat{\mathbf{e}}$ is the unit photon polarization vector, and $d \Omega$ is the differential sold angle. In dusty plasmas consisting of electrons, ions, and negatively charged dusty grains, the quasineutral condition ${ }^{14}$ at equilibrium is represented by

$$
n_{e 0}+Z_{d} n_{d 0}=n_{i 0}=n_{0}
$$

where $n_{j 0}(j=e, i, d)$ are, respectively, the equilibrium densities for electrons $(e)$, ions $(i)$, and dust grains $(d),-Z_{d}$ is the charge number of the dusty grains, and $n_{0}$ is the total plasma density. In the case of $\left|e \phi / k_{B} T_{j}\right| \ll 1 \quad(j=e, i)$, the Poisson's equation $^{12}$ in three-component dusty plasmas can be written as

$$
\nabla^{2} \phi=4 \pi e\left(n_{e}+Z_{d} n_{d}-n_{i}\right) \approx 4 \pi e n_{e 0} \frac{e \phi}{k_{B} T_{e}}\left(1+\frac{n_{0}}{n_{e 0}} \frac{T_{e}}{T_{i}}\right),
$$

where $\phi$ is the electrostatic potential in dusty plasmas, $k_{B}$ is the Boltzmann constant, and $T_{j}$ is the temperature of species 
$j$. The effective Debye length ${ }^{14} \lambda_{\text {eff }}$ in three-component dusty plasmas is then given by

$$
\lambda_{\mathrm{eff}}=\lambda_{D}\left(\frac{n_{e 0}}{n_{0}}+\frac{T_{e}}{T_{i}}\right)^{-1},
$$

where $\lambda_{D}\left(\equiv k_{B} T_{e} / 4 \pi n_{0} e^{2}\right)^{1 / 2}$ is the standard Debye length in electron-ion plasmas and the correction factor $\left(n_{e 0} / n_{0}\right.$ $\left.+T_{e} / T_{i}\right)$ stands for the influence of the dusty plasma on the Debye length. Since the correction factor is usually greater than unity, the effective Debye length in dusty plasmas is found to be greater than that in customary electron-ion plasmas. Hence, in spherical polar coordinates with their origin at the center of the dust grain, the interaction potential $V_{i-d}(r)$ between the ion with charge $z e$ and negatively charged dust grain with charge $-Z_{d} e$ in dusty plasmas is represented by the Yukawa form with the effective Debye length $\lambda_{\text {eff }}$,

$$
V_{i-d}(r)=-\frac{Z_{d} z e^{2}}{r} \exp \left(-r / \lambda_{\text {eff }}\right) .
$$

For the sake of simplicity, the dust grains are assumed to be spherical shapes throughout in this work. After some algebra, the Fourier transformation $\bar{V}_{i-d}(q)$ of the ion-dust grain interaction potential is then given by

$$
\begin{aligned}
\bar{V}_{i-d}(q)= & -Z_{d} z e^{2} \int \frac{d^{3} \mathbf{r}}{r} \exp \left(-r / \lambda_{\mathrm{eff}}-i \mathbf{q} \cdot \mathbf{r}\right) \\
= & -\frac{4 \pi Z_{d} z e^{2} a}{q} \frac{\exp \left(-a / \lambda_{\mathrm{eff}}\right)}{\left(a / \lambda_{\mathrm{eff}}\right)^{2}+(q a)^{2}} \\
& \times\left[\left(a / \lambda_{\mathrm{eff}}\right) \sin (q a)+(q a) \cos (q a)\right],
\end{aligned}
$$

where $a$ is the radius of the spherical dust grain. Hence, the ion-dust bremsstrahlung cross section in dusty plasmas is then given by

$$
\begin{aligned}
d^{2} \sigma_{b}= & \frac{16}{3} \frac{\alpha^{3} a_{0}^{2}}{\bar{E}_{0}} \frac{m_{e}}{m_{i}} Z_{d}^{2} z^{2} \exp \left(-2 \bar{\lambda}_{\text {eff }}^{-1}\right) \\
& \times\left(\frac{\bar{\lambda}_{\text {eff }}^{-1} \sin \bar{q}+\bar{q} \cos \bar{q}}{\bar{\lambda}_{\text {eff }}^{-2}+\bar{q}^{2}}\right)^{2} \bar{q} d \bar{q} \frac{d \omega}{\omega},
\end{aligned}
$$

where $a_{0}\left(=\hbar^{2} / m_{e} e^{2}\right)$ is the first Bohr radius of the hydrogen atom, $m_{e}$ is the mass of the electron, $\bar{E}_{0}\left(\equiv m_{i} v_{0}^{2} / 2 R y\right)$ is the scaled initial collision energy, $R y\left(=m_{e} e^{4} / 2 \hbar^{2} \approx 13.6 \mathrm{eV}\right)$ is the Rydberg constant, $\bar{q}(\equiv q a)$ is the scaled momentum transfer, and $\bar{\lambda}_{\text {eff }}\left(\equiv \lambda_{\text {eff }} / a\right)$ is the scaled effective Debye length. This expression of the bremsstrahlung cross section is reliable when the kinetic energy of the projectile ion is greater than the interaction energy between the ion and dust grain due to the prerequisite of the Born approximation. It has been also shown that the continuum spectrum due to the bremsstrahlung process would be investigated through the bremsstrahlung radiation cross section ${ }^{15}$ defined as $d^{2} \chi_{b} / d \bar{\varepsilon} d \bar{q} \equiv \hbar \omega\left(d \sigma_{b} / \hbar d \omega d \bar{q}\right)$, where $\bar{\varepsilon}(\equiv \varepsilon / R y)$ is the scaled photon energy and $\varepsilon(\equiv \hbar \omega)$ is the photon energy. After some mathematical manipulations, the ion-dust grain bremsstrahlung radiation cross section $d^{2} \chi_{b} / d \bar{\varepsilon}$ in threecomponent dusty plasmas is then obtained by the following analytic form:

$$
\begin{aligned}
& \frac{d^{2} \chi_{b}}{d \bar{\varepsilon}}=\frac{4}{3} \frac{\alpha^{3} a_{0}^{2}}{\bar{E}_{0}}\left(\eta Z_{d} z\right)^{2} \exp \left(-2 \xi^{-1} \bar{\lambda}_{D}^{-1}\right) \\
& \times \mid \ln \left\{\frac{1+\left[\bar{a} \eta \xi \bar{\lambda}_{D}\left(\sqrt{\bar{E}_{0}}+\sqrt{\bar{E}_{f}}\right)\right]^{2}}{1+\left[\bar{a} \eta \xi \bar{\lambda}_{D}\left(\sqrt{\bar{E}_{0}}-\sqrt{\bar{E}_{f}}\right)\right]^{2}}\right\} \\
& +\left(1+2 \xi^{-1} \bar{\lambda}_{D}^{-1}\right)\left[\cosh \left(2 \xi^{-1} \bar{\lambda}_{D}^{-1}\right)+\sinh \left(2 \xi^{-1} \bar{\lambda}_{D}^{-1}\right)\right] \\
& \times\left\{\sum _ { l = 1 } ^ { 2 } \left\{\mathrm{Ci}\left[(-1)^{l} 2 i \xi^{-1} \bar{\lambda}_{D}^{-1}+2 \bar{a} \eta\left(\sqrt{\bar{E}_{0}}+\sqrt{\bar{E}_{f}}\right)\right]\right.\right. \\
& \left.-\operatorname{Ci}\left[(-1)^{l} 2 i \xi^{-1} \bar{\lambda}_{D}^{-1}+2 \bar{a} \eta\left(\sqrt{\bar{E}_{0}}-\sqrt{\bar{E}_{f}}\right)\right]\right\} \\
& +i \sum_{l=1}^{2}\left\{\operatorname{Si}\left[2 i \xi^{-1} \bar{\lambda}_{D}^{-1}+(-1)^{l} 2 \bar{a} \eta\left(\sqrt{\bar{E}_{0}}+\sqrt{\bar{E}_{f}}\right)\right]\right. \\
& \left.-\mathrm{Si}\left[2 i \xi^{-1} \bar{\lambda}_{D}^{-1}+(-1)^{l} 2 \bar{a} \eta\left(\sqrt{\bar{E}_{0}}-\sqrt{\bar{E}_{f}}\right)\right]\right\} \\
& +\frac{2 \cos \left[\bar{a} \eta\left(\sqrt{\bar{E}_{0}}+\sqrt{\bar{E}_{f}}\right)\right]}{1+\left[\bar{a} \eta \xi \overline{\lambda_{D}}\left(\sqrt{\bar{E}_{0}}+\sqrt{\bar{E}_{f}}\right)\right]^{2}} \\
& -\frac{2 \cos \left[\bar{a} \eta\left(\sqrt{\bar{E}_{0}}-\sqrt{\bar{E}_{f}}\right)\right]}{1+\left[\bar{a} \eta \bar{\xi} \bar{\lambda}_{D}\left(\sqrt{\bar{E}_{0}}-\sqrt{\bar{E}_{f}}\right)\right]^{2}} \\
& -\frac{2 \bar{a} \eta \xi \bar{\lambda}_{D}\left(\sqrt{\bar{E}_{0}}+\sqrt{\overline{\bar{E}}_{f}}\right) \sin \left[\bar{a} \eta\left(\sqrt{\overline{\bar{E}}_{0}}+\sqrt{\overline{\bar{E}}_{f}}\right)\right]}{1+\left[\bar{a} \eta \xi \bar{\lambda}_{D}\left(\sqrt{\bar{E}_{0}}+\sqrt{\overline{\bar{E}}_{f}}\right)\right]^{2}} \\
& +\left.\frac{2 \bar{a} \eta \xi \bar{\lambda}_{D}\left(\sqrt{\bar{E}_{0}}-\sqrt{\bar{E}_{f}}\right) \sin \left[\bar{a} \eta\left(\sqrt{\bar{E}_{0}}-\sqrt{\bar{E}_{f}}\right)\right]}{1+\left[\bar{a} \eta \xi \bar{\lambda}_{D}\left(\sqrt{\bar{E}_{0}}-\sqrt{\bar{E}_{f}}\right)\right]^{2}}\right|^{2},
\end{aligned}
$$

where $\eta \equiv \sqrt{m_{e} / m_{i}}, \bar{E}_{f}\left(\equiv \bar{E}_{0}-\bar{\varepsilon}\right)$ is the scaled final projectile energy, $\bar{a}\left(\equiv a / a_{0}\right)$ is the scaled radius of the dust grain, $\bar{\lambda}_{D}$ $\equiv \lambda_{D} / a, \quad \xi \equiv\left(n_{e 0} / n_{0}+T_{e} / T_{i}\right)^{-1}$, and $\operatorname{Ci}(x)\left(=-\int_{x}^{\infty} d t \cos t / t\right)$ and $\operatorname{Si}(x)\left(=\int_{0}^{x} d t \sin t / t\right)$ are the cosine and sine integrals, ${ }^{16}$ respectively. Very recently, an excellent discussion ${ }^{17}$ on the additional part of the electrostatic potential in dusty plasmas is given due to the ion absorption by the dust grain and ion-neutral collisions. It has been found that the electrostatic potential would be represented only by the standard DebyeHückel form if there is no ion flux on the surface of the dust grain, i.e., nonabsorbing dust grains. In this work, we just retain only the Debye-Hückel form of the interaction potential since we consider the bremsstrahlung process due to the scattering of the ion by the nonabsorbing dust grain. However, if the ion flux exists on the dust grain, the attractive part of the potential has to be included in the ion-dust bremsstrahlung process. Hence, it would be expected that the additional part of the interaction potential suppresses the ion-dust grain bremsstrahlung spectrum obtained by the Yukawa-type Debye-Hückel potential. Since the Born parameter

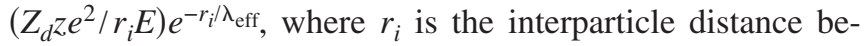
tween the ion and dust grain, for the ion-dust interaction in typical circumstances of dusty plasmas is usually less than unity, the Born approximation would be quite reliable to investigate the high energy ion-dust bremsstrahlung process in dusty plasmas. Very recently, the accurate form of the effec- 


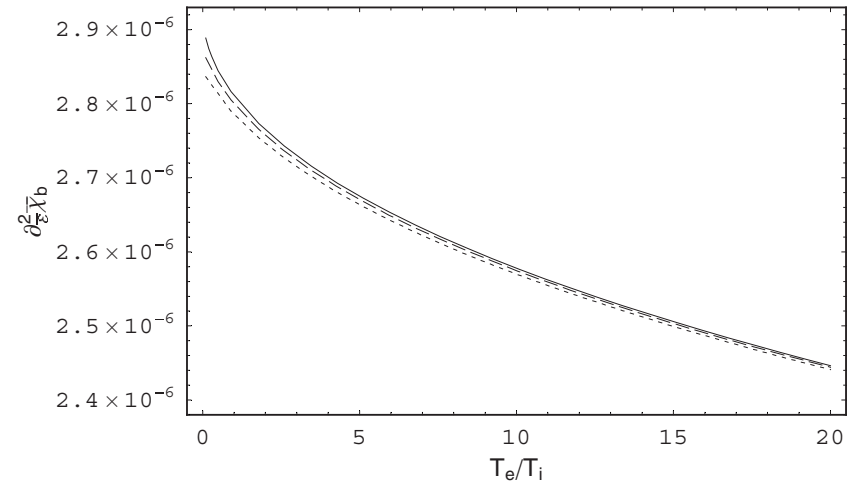

FIG. 1. The scaled ion-dust grain bremsstrahlung radiation cross section $\partial_{\bar{\varepsilon}}^{2} \bar{\chi}_{b}$ as a function of the temperature ratio $T_{e} / T_{i}$ for $\bar{E}_{0}=5, \bar{\varepsilon}=2, T_{e}=5$ $\times 10^{3} \mathrm{~K}, n_{0}=10^{10} \mathrm{~cm}^{-3}$, and $a=9.7 \times 10^{-5} \mathrm{~cm}$. The solid line represents the case of $n_{e 0} / n_{0}=0$. The dashed line represents the case of $n_{e 0} / n_{0}=0.2$. The dotted line represents the case of $n_{e 0} / n_{0}=0.5$.

tive polarization interaction potential ${ }^{18}$ has been given in partially ionized dense plasmas, taking into account the quantum mechanical effects of diffraction and symmetry of particles and plasma screening effects. However, the ionatom polarization bremsstrahlung process is neglected in this work since the dust plasma is assumed to be completely ionized. For typical circumstances of dusty plasmas, it has been shown that $Z_{d} \approx 100-1000, a \approx 0.01-1 \mu \mathrm{m}$, and $\lambda_{D} / a \approx 5-100{ }^{8}$

In order to specifically investigate the effects of electron temperature and density on the ion-dust bremsstrahlung process in a dusty plasma, we set $Z_{d}=200, a=0.1 \mu \mathrm{m}, \lambda_{D} / a$ $=50, \quad m_{i} / m_{e}=1840$, and $z=1$. Figure 1 shows the scaled bremsstrahlung radiation cross section $\partial_{\bar{\varepsilon}}^{2} \bar{\chi}_{b}[$ $\left.\equiv\left(d^{2} \chi_{b} / d \bar{\varepsilon} / \pi a_{0}^{2}\right)\right]$ in units of $\pi a_{0}^{2}$ for the interaction of the ion with the negatively charged dust grain in dusty plasmas as a function of the temperature ratio $T_{e} / T_{i}$ for various values of the density ratio $n_{e 0} / n_{0}$. In addition, Fig. 2 represents the scaled bremsstrahlung radiation cross section $\partial_{\bar{\varepsilon}}^{2} \bar{\chi}_{b}$ as a function of the density ratio $n_{e 0} / n_{0}$ for various values of the

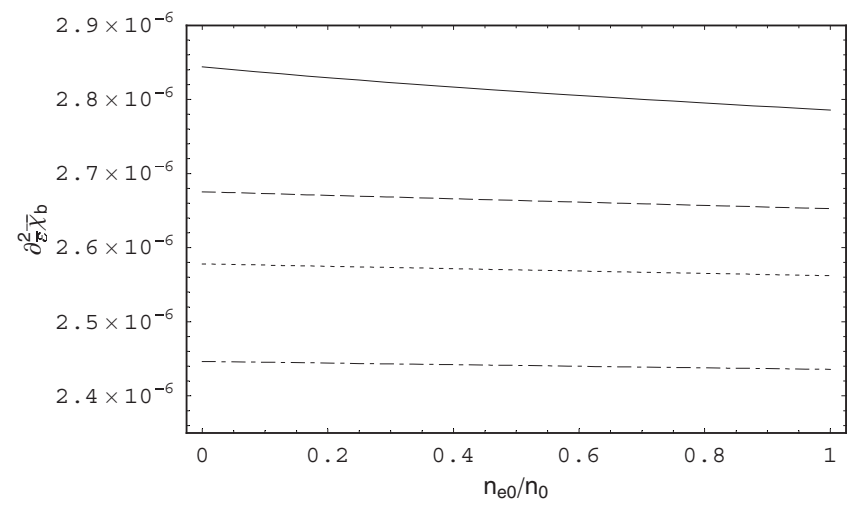

FIG. 2. The scaled ion-dust grain bremsstrahlung radiation cross section $\partial_{\bar{\varepsilon}}^{2} \bar{\chi}_{b}$ as a function of the temperature ratio $n_{e 0} / n_{0}$ for $\bar{E}_{0}=5, \bar{\varepsilon}=2, T_{e}=5$ $\times 10^{3} \mathrm{~K}, n_{0}=10^{10} \mathrm{~cm}^{-3}$, and $a=9.7 \times 10^{-5} \mathrm{~cm}$. The solid line represents the case of $T_{e} / T_{i}=0.5$. The dashed line represents the case of $T_{e} / T_{i}=5$. The dotted line represents the case of $T_{e} / T_{i}=10$. The dot-dashed line represents the case of $T_{e} / T_{i}=20$. temperature ratio $T_{e} / T_{i}$. As shown in these figures, it is found that the ion-dust bremsstrahlung radiation cross section decreases with an increase in the ratio of the electron temperature to the ion temperature in a dusty plasma. It is also shown that the electron density suppresses the ion-dust bremsstrahlung radiation cross section. It is interesting to note that the effect of electron density on the bremsstrahlung radiation cross section diminishes with an increase in the electron temperature since the high energy electrons can be readily repelled by the negatively charged dusty grains in dusty plasmas. ${ }^{12}$ The effect of electron density is found to be important for low-temperature ratios. It is also found that the effect of electron temperature on the bremsstrahlung radiation cross section is found to be more significant than the effect of electron density in dusty plasmas. Hence, we have found that the effects of electron temperature and density play important roles in the ion-dust grain bremsstrahlung process in dusty plasmas containing electrons, ions, and negatively charged dust grains. These results would provide useful information on the ion-dust bremsstrahlung emission spectrum and also the radiation due to the interaction between dust particle chains and streaming ions in the plasma sheath in dusty plasmas.

The authors acknowledge Professor T. Kato for providing us useful data and references for dust particles in LHD plasmas. One of the authors (Y.D.J.) also gratefully acknowledges the Director-General Professor O. Motojima, Director Professor M. Sato, Director Professor Y. Hirooka, and Dr. D. Kato for warm hospitality and support while visiting the National Institute for Fusion Science (NIFS) in Japan as a longterm visiting professor. The authors are also grateful to NIFS for supporting the research. This work was done while Y.D.J. was visiting NIFS.

${ }^{1}$ H. A. Bethe and E. E. Salpeter, Quantum Mechanics of One- and TwoElectron Atoms (Springer, Berlin, 1957).

${ }^{2}$ G. Bekefi, Radiation Processes in Plasmas (Wiley, New York, 1966).

${ }^{3}$ H. Totsuji, Phys. Rev. A 32, 3005 (1985).

${ }^{4}$ V. P. Shevelko, Atoms and Their Spectroscopic Properties (Springer, Berlin, 1997).

${ }^{5}$ H. F. Beyer, H.-J. Kluge, and V. P. Shevelko, X-Ray Radiation of Highly Charged Ions (Springer, Berlin, 1997).

${ }^{6} \mathrm{~V}$. Shevelko and H. Tawara, Atomic Multielectron Processes (Springer, Berlin, 1998).

${ }^{7}$ H. F. Beyer and V. P. Shevelko, Introduction to the Physics of Highly Charged Ions (Institute of Physics Publishing, Bristol, 2003).

${ }^{8}$ D. A. Mendis and M. Rosenberg, Annu. Rev. Astron. Astrophys. 32, 419 (1994).

${ }^{9}$ A. Bouchoule, Dusty Plasmas: Physics, Chemistry and Technological Impacts in Plasma Processing (Wiley, Chichester, 1999).

${ }^{10}$ P. K. Shukla and A. A. Mamum, Introduction to Dusty Plasma Physics (Institute of Physics Publishing, Bristol, 2002).

${ }^{11}$ T. S. Ramazanov et al., Phys. Plasmas 15, 053704 (2008).

${ }^{12}$ S. A. Maiorov et al., Phys. Plasmas 15, 093701 (2008).

${ }^{13}$ R. J. Gould, Electromagnetic Processes (Princeton University Press, Princeton, 2006).

${ }^{14}$ S. Vidhya Lakshmi et al., Astrophys. Space Sci. 209, 213 (1993).

${ }^{15}$ J. D. Jackson, Classical Electrodynamics (Wiley, New York, 1962).

${ }^{16} \mathrm{G}$. Arfken, Mathematical Methods for Physicists (Academic, New York, 1966).

${ }^{17}$ S. A. Khrapak and G. E. Morfill, Phys. Plasmas 15, 084502 (2008).

${ }^{18}$ T. S. Ramazanov et al., Phys. Plasmas 12, 092702 (2005). 